\title{
Recombinant oncolytic Newcastle disease virus displays antitumor activities in anaplastic thyroid cancer cells
}

Ke Jiang ${ }^{1 \dagger}$, Cuiping Song ${ }^{2 \dagger}$, Lingkai Kong ${ }^{1 \dagger}$, Lulu Hu', Guibin Lin ${ }^{5}$, Tian Ye ${ }^{1}$, Gang Yao ${ }^{1}$, Yupeng Wang ${ }^{6}$, Haibo Chen ${ }^{1}$, Wei Cheng ${ }^{1}$, Martin P. Barr ${ }^{3}$, Quentin Liu', Guirong Zhang ${ }^{4^{*}}$, Chan Ding ${ }^{2^{*}}$ and Songshu Meng ${ }^{{ }^{*}}$

\begin{abstract}
Background: Anaplastic thyroid cancer (ATC) is one of the most aggressive of all solid tumors for which no effective therapies are currently available. Oncolytic Newcastle disease virus (NDV) has shown the potential to induce oncolytic cell death in a variety of cancer cells of diverse origins. However, whether oncolytic NDV displays antitumor effects in ATC remains to be investigated. We have previously shown that the oncolytic NDV strain FMW (NDV/FMW) induces oncolytic cell death in several cancer types. In the present study, we investigated the oncolytic effects of NDV/FMW in ATC.
\end{abstract}

Methods: In this study, a recombinant NDV expressing green fluorescent protein (GFP) was generated using an NDV reverse genetics system. The resulting virus was named after rFMW/GFP and the GFP expression in infected cells was demonstrated by direct fluorescence and immunoblotting. Viral replication was evaluated by end-point dilution assay in DF-1 cell lines. Oncolytic effects were examined by biochemical and morphological experiments in cultural ATC cells and in mouse models.

Results: rFMW/GFP replicated robustly in ATC cells as did its parent virus (NDV/FMW) while the expression of GFP protein was detected in lungs and spleen of mice intravenously injected with rFMW/GFP. We further showed that rFMW/GFP infection substantially increased early and late apoptosis in the ATC cell lines, THJ-16 T and THJ-29 T and increased caspase-3 processing and Poly (ADP-ribose) polymerase (PARP) cleavage in ATC cells as assessed by immunoblotting. In addition, rFMW/GFP induced lyses of spheroids derived from ATC cells in three-dimensional (3D) cultures. We further demonstrated that rFMW/GFP infection resulted in the activation of p38 MAPK signaling, but not Erk1/2 or JNK, in THJ-16 T and THJ-29 T cells. Notably, inhibition of p38 MAPK activity by SB203580 decreased rFMW/GFP-induced cleavage of caspase-3 and PARP in THJ-16 T and THJ-29 T cells. Finally, both rFMW/GFP and its parent virus inhibited tumor growth in mice bearing THJ-16 T derived tumors.

(Continued on next page)

\footnotetext{
*Correspondence: zhang.lth@163.com; shoveldeen@shvri.ac.cn; ssmeng@dmu.edu.cn

${ }^{\dagger}$ Ke Jiang, Cuiping Song and Lingkai Kong contributed equally to this work. ${ }^{4}$ Central laboratory, Liaoning Cancer Hospital and Institute, Cancer Hospital of China Medical University, 44 Xiaoheyan Road, Shenyang 110042, China ${ }^{2}$ Department of Avian Infectious Diseases, Shanghai Veterinary Research Institute, Chinese Academy of Agricultural Sciences, 518 Ziyue Road, Shanghai 200241, China

${ }^{1}$ Institute of Cancer Stem Cell, Dalian Medical University Cancer Center,

Room 415, 9 Lvshun Road South, Dalian 116044, China

Full list of author information is available at the end of the article
}

(c) The Author(s). 2018 Open Access This article is distributed under the terms of the Creative Commons Attribution 4.0 International License (http://creativecommons.org/licenses/by/4.0/), which permits unrestricted use, distribution, and reproduction in any medium, provided you give appropriate credit to the original author(s) and the source, provide a link to the Creative Commons license, and indicate if changes were made. The Creative Commons Public Domain Dedication waiver (http://creativecommons.org/publicdomain/zero/1.0/) applies to the data made available in this article, unless otherwise stated. 
(Continued from previous page)

Conclusion: Taken together, these data indicate that both the recombinant reporter virus rFMW/GFP and its parent virus NDV/FMW, display oncolytic activities in ATC cells in vitro and in vivo and suggest that oncolytic NDV may have potential as a novel therapeutic strategy for ATC.

Keywords: Anaplastic thyroid cancer (ATC), Newcastle disease virus (NDV), p38 MAPK, Green fluorescent protein (GFP), Apoptosis,

\section{Background}

Anaplastic thyroid cancer (ATC) is the most aggressive type among thyroid cancers, accounting for a significant portion of thyroid cancer death [1]. Current treatments for ATC patients such as surgery, radiotherapy and chemotherapy have no effect in increasing patients' survival [2]. Therefore, the development of novel therapeutic approaches for ATC is urgently needed.

Oncolytic viruses (OVs) are naturally occurring or engineered viruses that selectively infect and replicate in cancer cells, triggering direct oncolysis. Several preclinical studies have demonstrated that OV-based therapy is effective in the treatment of ATC [3]. A series of studies by Portella \& colleagues has shown that oncolytic adenovirus strains dl1520 (Onyx-015) and d1922-947, alone or in combination with rationally designed molecularly-targeted drugs, displayed antitumor activities in ATC cells and in in vivo mouse models [4-9]. Similarly, the adenovirus strain, ONYX-411, induced cell death in ATC cell lines and suppressed the growth of xenograft tumors in nude mice [10]. In addition to oncolytic adenoviruses, oncolytic vaccina viruses also displayed antitumor activities in ATC cells and in xenograft models $[11,12]$. Wong et al. investigated the oncolytic effects of oncolytic vaccina virus strains NV1023 and GLV-1 h68 in ATC in the preclinical setting [13-16]. Other OVs such as measles virus has also been demonstrated to induce cytotoxicity in ATC cells [17]. Together, these studies strongly indicate that OVs hold promise for the treatment of patients with ATC.

Newcastle disease virus (NDV) is a member of the Avulavirus genus in the Paramyxoviridae family. Naturally occurring strains of NDV and recombinant NDV expressing immunoregulatory factors have demonstrated the potential to kill cancer cells of diverse origin in both preclinical and clinical studies $[18,19]$. However whether oncolytic NDV displays antitumor effects in ATC remains to be investigated. We have previously shown that either naturally occurring or recombinant oncolytic NDV expressing apoptin triggers oncolytic cell death in lung and liver tumor cell lines and tumor-bearing mice [20-24]. The aim of the present study was to determine the oncolytic efficacy of NDV using a recombinant NDV-expressing GFP protein in ATC cell lines and mouse model. To better understand oncolytic NDV infection process in cancer cells, we generated a recombinant NDV expressing the green fluorescent protein (GFP). We evaluated the efficacy of the recombinant NDV in ATC cell lines and in mouse models. Our results show that the GFP-expressing reporter NDV, exhibits potent oncolytic activities in ATC cell lines and in a mouse model of thyroid cancer.

\section{Methods}

Cells, viruses and regent

Chicken embryo fibroblast cell line, DF1 (cat no. GNO30), was obtained and authenticated by the Cell Bank of the Chinese Academy of Science (Shanghai, China). Cells were maintained in DMEM supplemented with $10 \%$ fetal bovine serum (FBS). THJ-16 T and THJ-29 T cells were kindly provided by the Mayo Foundation for Medical Education and Research to Dr. Quentin Liu [25]. THJ-16 T cells were cultured in RPMI-1640 containing 5\% FBS, $10 \mathrm{mM}$ HEPES (Thermo Fisher) and $1 \mathrm{mM}$ sodium pyruvate (Thermo Fisher). THJ-29 T cells were cultured in RPMI-1640 containing 5\% FBS and $1 \mathrm{mM}$ sodium pyruvate. A virulent strain of NDV/FMW (GenBank accession number: GU564399) was prepared as reported previously [20]. SB203580, a specific p38 inhibitor, was purchased from Selleckchem which was prepared with dimethyl sulfoxide (DMSO) and stored at $-20^{\circ} \mathrm{C}$.

\section{Construction of GFP-labelled recombinant NDV/FMW}

The construction of the recombinant NDV/FMW expressing GFP was performed essentially as described in our previous study for the generation of the recombinant NDV/FMW expressing apoptin [24]. To construct rFMW-GFP, a GFP-labeled fragment flanked by the appropriate NDV-specific RNA transcriptional signals was inserted into the ApaI site created between the $\mathrm{P}$ and $\mathrm{M}$ genes of pT7NDV/FMW. The resulted plasmid was named as rFMW-GFP and sequencing verified. Viruses were rescued from complementary cDNA using methods described previously [24]. The resultant recombinant virus, rFMW/ GFP was prepared, stored and titered as previously described [20-22, 24].

\section{Live cell imaging}

THJ-16 $\mathrm{T}$ and THJ-29 $\mathrm{T}$ cells were cultured in 6-well plates and infected with NDV/FMW or rFMW/GFP at a multiplicity of infection (MOI) of 10. Cells were 
observed using fluorescence microscopy (Olympus IX81). Live cell imaging of bright-field and fluorescence was recorded at $24 \mathrm{~h}$ post-infection.

\section{Immunofluorescence assay}

THJ-16 T and THJ-29 T cells for immunofluorescence assay were seeded on coverslips (NEST, 801008) in 24 wells plate and fixed in $4 \%$ paraformaldehyde (PFA) for $30 \mathrm{~min}$, then the cells were permeabilized in $0.2 \%$ Triton $\mathrm{X}-100$ for $15 \mathrm{~min}$. Non-specific binding sites were blocked by incubation with 3\% Bovine Serum Albumin (BSA) for $60 \mathrm{~min}$. Cells were then incubated with primary anti-HN antibody (1:50) overnight at $4{ }^{\circ} \mathrm{C}$. After washed, secondary antibodies (1:1000) were added to appropriate wells. After $60 \mathrm{~min}$, Nuclei were stained with DAPI $(5 \mu \mathrm{g} / \mathrm{mL}$, Sigma) in PBS. Images were acquired using a confocal microscope (Leica TCS SP $5 \times$ ) and images were captured with a camera controlled. Images from each experiment were acquired using the same exposure time during the same imaging session.

\section{Immunoblot assay}

THJ-16 T and THJ-29 T cells were seeded in $60-\mathrm{mm}$ dishes and infected with vehicle or $\mathrm{rFMW/GFP}$ at 10 MOI. Cells were harvested using scraper and lysed in lysis buffer (Roche, USA) at 6,12 or $24 \mathrm{~h}$. Cell samples were loaded and separated by 10 or $15 \%$ SDS-PAGE and subsequently transferred to nitrocellulose membranes (Applygen Technologies Inc. Beijing, China) using a transblot turbo system. Membrane was blocked with $5 \%$ milk diluted in TBST buffer $(0.05 \%$ Tween-20) for $3 \mathrm{~h}$ and incubated with primary antibody at $4{ }^{\circ} \mathrm{C}$ overnight. The antibodies for GFP-tag (1:10000, Sigma, SAB4301138), HN (1:500, Santa cruz, SC-53562), $\beta$-actin (1:10000, Sigma, A1978), caspase-3 (1:1000, Cell signaling technology, 9662S), PARP (1:1000, Cell signaling technology, 9532S), phospho-p38 (1:1000, Cell signaling technology, 9215S), total p38 (1:1000, cell signaling technology, 9212S), phospho-Erk1/2 (1:8000, Promega, V803A), total Erk1/2 (1:8000, Promega, V114A), phospho-JNK (1:2000, Cell signaling technology, 9251S) and total JNK (1:1000, Cell signaling technology, 9253S) were used. After washed three times with TBST, the membranes were incubated with horseradish peroxidase-conjugated secondary antibody (1:10000, Invitrogen, USA) for $1 \mathrm{~h}$ at room temperature with continuous rocking. The blots were detected using ECL Western Blot Substrate kit (Thermo Fisher, USA) [20].

\section{GFP expression in vivo}

rFMW/GFP $\left(1 \times 10^{7}\right.$ TCID50 per dose $)$ was injected intravenously (i.v) into BALB/c mice. To assess GFP expression in organs, mice were euthanized $24 \mathrm{~h}$ following virus injection. Heart, liver, spleen, lungs and kidneys were harvested. Cells were lysed and GFP protein was visualized by IB.

\section{Viral titer assay}

DF1 cells were seeded in 96-well plates and then infected with 10-fold serially diluted viruses. Viral titer was measured by end-point dilution assay (50\% tissue culture infective dose (TCID50]/ml) and the TCID50 was calculated by the method of Reed and Muench (Reed and Muench, 1938).

\section{Cell viability assay}

Cell viability was quantified using the 3-(4,5-dimethylthiazol-2-yl)-2,5-diphenyltetrazolium bromide (MTT) assay based on the formation of formazan crystals from tetrazolium by living/metabolically active cells. THJ-16 T and $\mathrm{THJ}-29 \mathrm{~T}$ cells were seeded in 96 -wells plates $(5000$ cells/ well), and then the cells were vehicle-infected or infected with varying MOI of $\mathrm{rFMW/GFP}(0.01,0.1,1$, and 10) for $24,48,72 \mathrm{~h}$. Cell growth inhibition was determined as previously described [22].

\section{Spheroid formation}

THJ-16 T and THJ-29 T spheroids were prepared from monolayer cells which were trypsinised and plated in ultra-low attachment 96 -well plates (1000 cells/well). The cells were containing in serum-free DMEM/F12 medium supplemented with $10 \mathrm{ng} / \mathrm{ml}$ basic fibroblast growth factor (bFGF), $20 \mathrm{ng} / \mathrm{ml}$ epidermal growth factor (EGF) and $1 \times$ B27. After 7 days, the propagated spheroid bodies were observed and counted by light microscope.

\section{In vivo oncolysis}

Female age-matched (6 weeks old) nude mice were housed in specific pathogen-free (SPF) conditions. THJ-16 T cell suspension $\left(5 \times 10^{6}\right.$ cells in $100 \mu \mathrm{L}$ PBS/ mouse) was injected subcutaneously in the right flank to induce tumor development. When tumors reached an average volume of $200 \mathrm{~mm}^{3}$, the rFMW/GFP treatments were initiated by intratumoral injection. Mice were randomly divided into two groups (eight mice per group): (a) vehicle control, (b) intratumoral administration with rFMW/GFP $\left(1 \times 10^{7}\right.$ TCID50 per dose $)$. Mice were injected three times weekly. After 1 week, four mice (of eight) were euthanized to make into slices. The slices were subjected to either hematoxylin-eosin (H\&E) staining or terminal deoxynucleotidyl transferase dUTP nick end labeling (TUNEL) assay as previously described [24]. The total proteins of tumor tissue samples were harvested from the other four mice in each group to test GFP and HN expression by immunoblot assay.

For the in vivo oncolysis study of growth curve, ten mice were included in each group ((a) vehicle control, 
(b) intratumoral administration with rFMW/GFP $(1 \times$ $10^{7}$ TCID50 per dose), (c) intratumoral administration with NDV/FMW $\left(1 \times 10^{7}\right.$ TCID50 per dose), was treated for 3 weeks. Tumor growth was monitored at 5-day intervals for 50 days using and volume was determined with digital caliper according to the formula: volume $=($ greatest diameter $) \times(\text { smallest diameter })^{2} / 2$. Euthanasia: Treat mice in an inhalation anesthesia machine (Shanghai Biowill Co., LTD, Model: BW-AM503) with $5 \%$ isoflurane (Sigma, cat no. Y0000858); $2.4 \mathrm{~L} / \mathrm{min}$ $\mathrm{N} 2 \mathrm{O} ; 1.2 \mathrm{~L} / \mathrm{min} \mathrm{O} 2$. Observe the mice. (The depth of anesthetization is sufficient when the following vital criteria are reached: regular spontaneous breathing. No reflex after setting of pain stimuli between toes, and no response to pain.) Carotid after anesthesia mice was killed off. The animals were tested in a biosafety cabinet of the SPF laboratory animal center of the Dalian Medical University (Dalian, China), complying with the national guidelines for the care and use of laboratory animals and were approved by the experimental animal ethics committee at Dalian Medical University.

\section{Statistical analysis}

For all experiments, statistical analysis was first performed using a one-way analysis of variance (ANOVA) to determine statistical significance between groups for each endpoint assessed. Multiple comparisons between treatment groups and controls were evaluated using Dunnett's LSD test. To assess the in vivo oncolytic effects, statistical significance between groups was calculated using the LSD post-test and SPSS 11.0 software (SPSS Inc., Chicago, IL, USA). $p$-values $<0.05$ was considered statistically significant.

\section{Results}

\section{Construction of recombinant NDV expressing GFP}

We have previously shown that the NDV strain FMW (NDV/FMW) exhibits strong oncolytic activity in vitro and in vivo [20-23]. To better monitor the NDV/FMW infection process in cancer cells, we generated recombinant NDV carrying the GFP reporter gene (Fig. 1a). The construction of the recombinant NDV/FMW expressing GFP (hereafter as rFMW/GFP) was performed utilizing the reverse genetics rescue system, which was employed in our previous study for the generation of the recombinant NDV/FMW expressing apoptin [24].

GFP expression in rFMW/GFP-infected ATC cells (THJ-16 T and THJ-29 T) was demonstrated by direct fluorescence and immunoblotting (Fig. $1 \mathrm{~b}$ and c). The expression of $\mathrm{HN}$ protein was detected in NDV/ FMW-infected cells (Fig. 1c). Importantly, detection of GFP was accompanied by the expression of $\mathrm{HN}$ protein

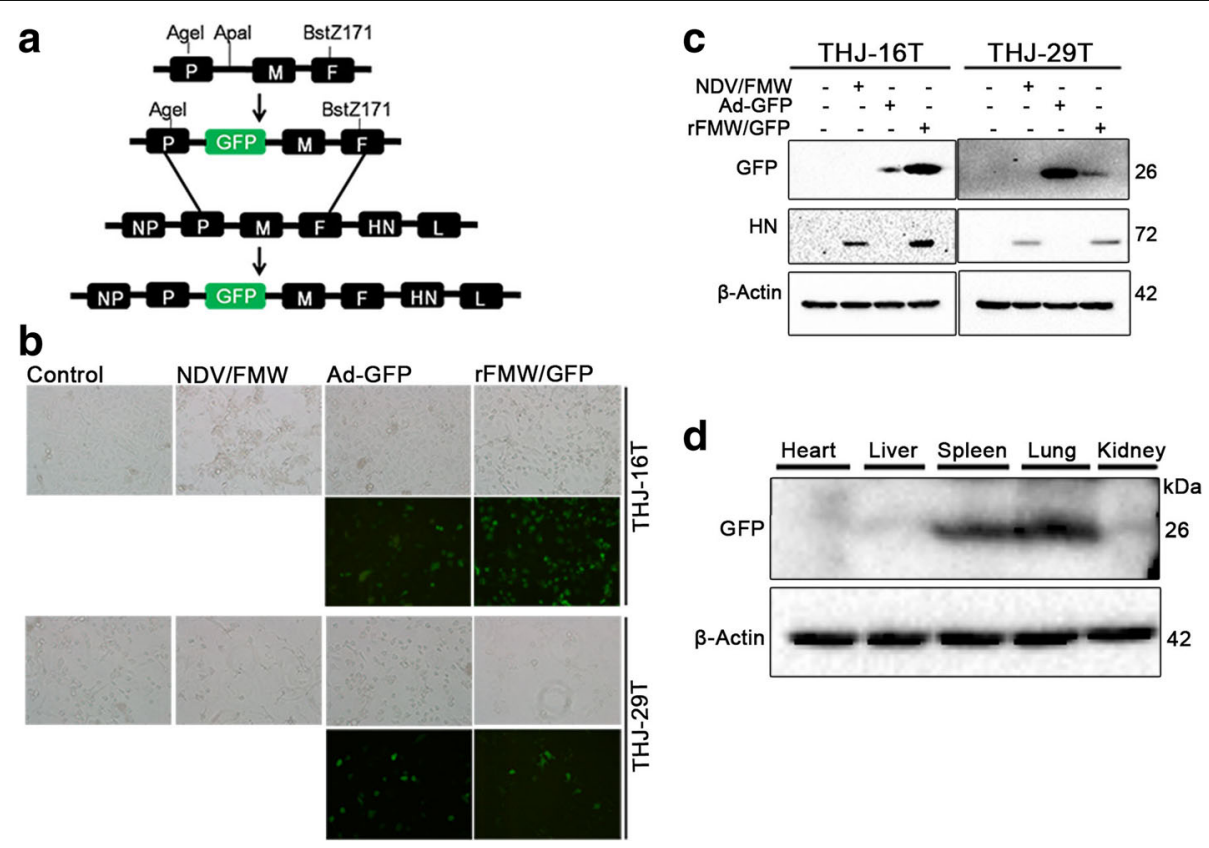

Fig. 1 Construction and identification of the recombinant rFMW/GFP. a PCR amplification of the DNA construct using Apal-tagged primers was carried out and subsequently introduced into the Anti-sense cDNA of the NDV strain, FMW. b THJ-16 T and THJ-29 T cells were infected with vehicle, NDV/FMW (10 MOI), adenovirus-GFP-Tag or rFMW/GFP (10 MOI) and imaged by fluorescence microscopy. Live cell imaging using bright-field and fluorescence microscopy was recorded at $24 \mathrm{~h}$ post-infection. c Protein levels of GFP-tagged and HN proteins were analyzed by immunoblotting (IB). d rFMW/GFP $\left(1 \times 10^{7}\right.$ TCID50 per dose) was i.v. injected into BALB/c mice. Mice were sacrificed $24 \mathrm{~h}$ post virus injection. Heart, liver, spleen, lungs and kidneys were harvested and GFP protein expression was assessed by IB. $\beta$-actin was used as a control for equal loading. All IB experiments were performed twice 
in rFMW/GFP-infected cells (Fig. 1c), indicating the replication of rFMW/GFP in infected cells. In addition, the stability of the recombinant virus was evaluated by inoculating rFMW/GFP into SPF embryonated chicken eggs after five serial passages. GFP insertion was subsequently confirmed by RT-PCR assay (data not shown).

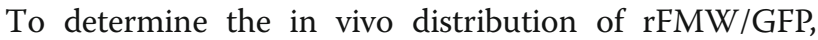
expression of GFP protein in organs from mice intravenously injected with rFMW/GFP was analyzed by immunoblotting. GFP protein was detected in spleen and lung (Fig. 1d), indicating the tissue tropism of rFMW/GFP and demonstrates the successful construction of a recombinant oncolytic NDV expressing GFP.

\section{Replication characteristics of rFMW/GFP}

To investigate whether GFP insertion caused any replication defect in NDV/FMW, the growth characteristics of $\mathrm{rFMW/GFP}$ and its parent virus were evaluated in a single-step growth cycle in DF-1 cell lines. The insertion of GFP into the NDV/FMW genome did not significantly influence viral replication kinetics and total viral yield during the $72 \mathrm{~h}$ post-infection (hpi) period compared to the parental NDV/FMW strain (Fig. 2a). However, detection of GFP was accompanied by the expression of $\mathrm{HN}$ protein in $\mathrm{rFMW} / \mathrm{GFP}$-infected $\mathrm{THJ}-16 \mathrm{~T}$ and $\mathrm{THJ}-29 \mathrm{~T}$ cells by confocal microscopy
(Fig. 2b), indicating the successful replication of rFMW/ GFP in the ATC cell lines tested.

\section{Oncolytic activity of rFMW/GFP in ATC cell lines using 2D} and $3 \mathrm{D}$ cultures

We proceeded to determine whether $\mathrm{rFMW/GFP}$ induced growth inhibition in ATC cell lines. rFMW/GFP infection induced a significant reduction in viability of THJ-16 $\mathrm{T}$ and $\mathrm{THJ}-29 \mathrm{~T}$ cell lines in a time and concentration-dependent manner compared to mock infections (Fig. 3a). Similar results were obtained in parent NDV/FMW-infected THJ-16 T and THJ-29 T cells (data not shown). To examine whether the rFMW/ GFP-induced growth inhibition of ATC cells was due to apoptosis, caspase- 3 and Poly (ADP-ribose) polymerase (PARP) cleavage, two classical markers of apoptosis, was demonstrated in rFMW/GFP-treated THJ-16 $\mathrm{T}$ and THJ-29 T cells at $12 \mathrm{hpi}$ as assessed by immunoblotting (Fig. 3b). Together, these data indicated that rFMW/GFP induced apoptosis in ATC cells.

Given that tumor spheroids are considered a useful in vitro model to mimic the biological properties of tumors, we examined whether the effects of rFMW/GFP on spheroids derived from ATC cells in a threedimensional (3D) culture system. Upon rFMW/GFP infection at an MOI of 10 for $48 \mathrm{~h}, \mathrm{rFMW} / \mathrm{GFP}$-infected a

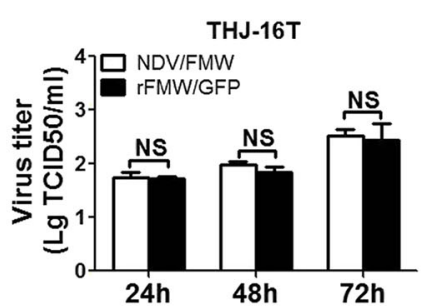

THJ-29T

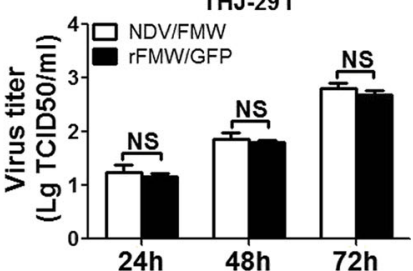

\section{b}

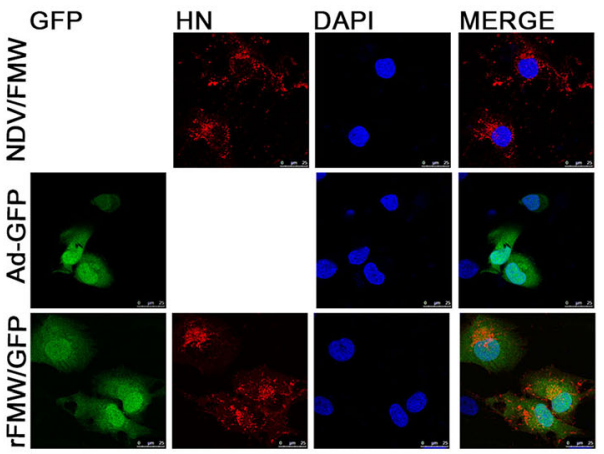

THJ-16T

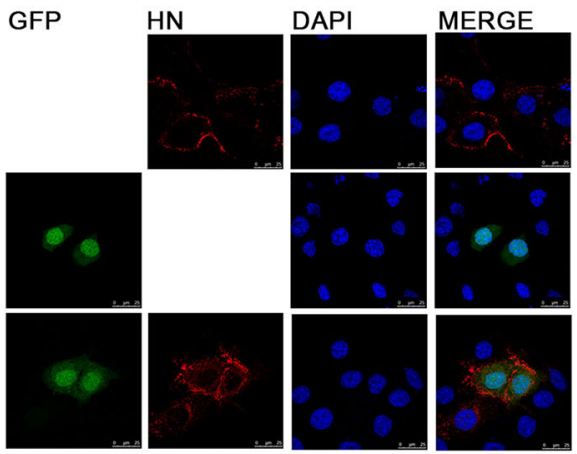

THJ-29T

Fig. 2 Replication characteristics of rFMW/GFP. a THJ-16 T and THJ-29 T cells were infected with NDV/FMW (0.01 MOI) or rFMW/GFP (0.01 MOI) for 24,48 and $72 \mathrm{~h}$ respectively. Virus yield was determined at different intervals. Each assay was repeated three times. Data are presented as the mean \pm SD for three independent experiments. $\mathbf{b}$ THJ-16 T and THJ-29 T cells were infected with NDV/FMW (10 MOI), Adenovirus-GFP-Tag or rFMW/GFP (10 MOI) for $24 \mathrm{~h}$, stained with rabbit anti-HN polyclonal antibody and visualized by confocal microscopy using three channels (405, 488 and $643 \mathrm{~nm})$. DAPI was used for nuclear staining 

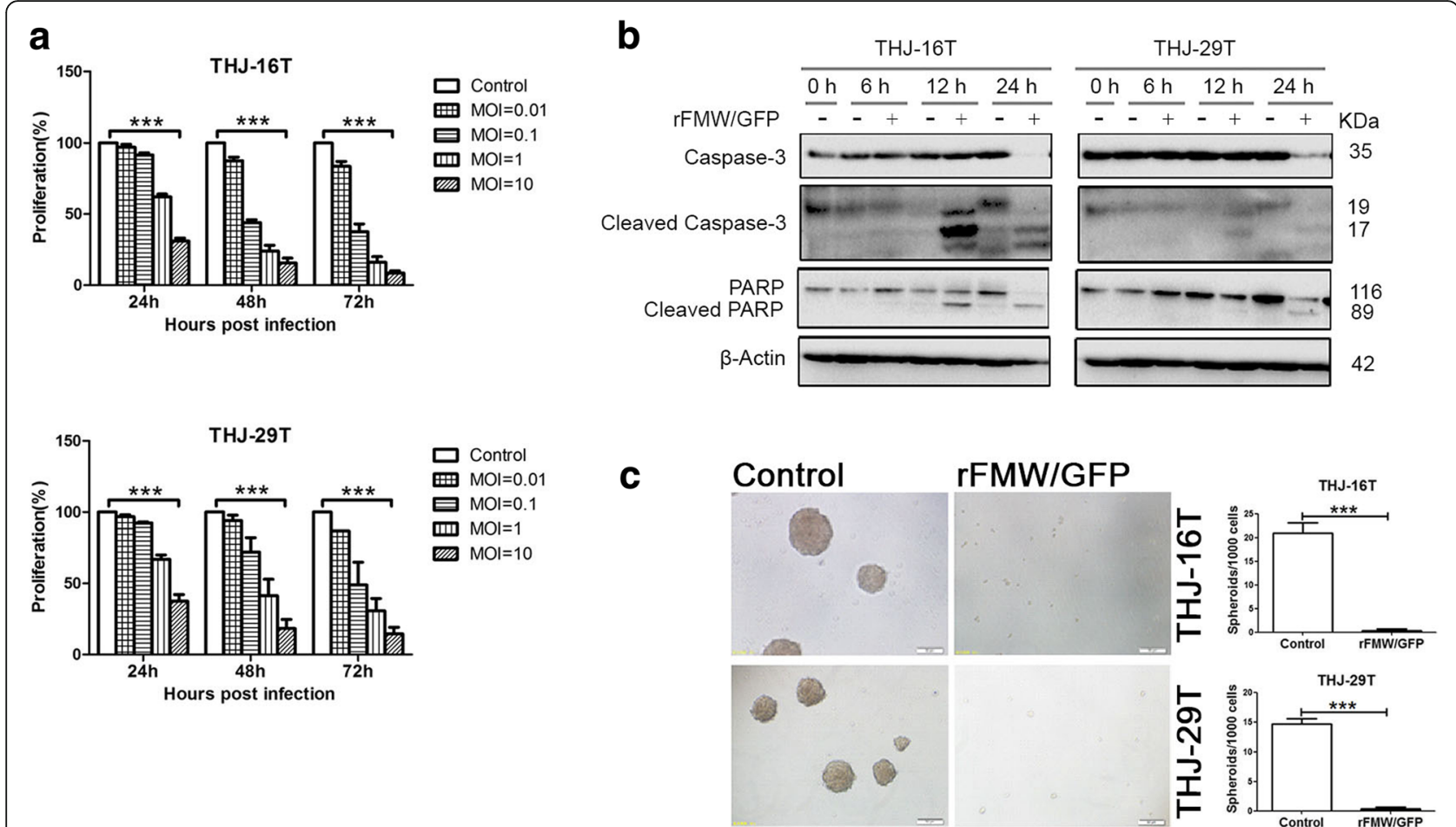

Fig. 3 Oncolytic activity exhibited by rFMW/GFP against ATC cells in both 2D and 3D cultures. a THJ-16 T and THJ-29 T cells were vehicle-infected or infected with varying MOI of rFMW/GFP $(0.01,0.1,1$ and 10$)$ for $24,48,72 \mathrm{~h}$ respectively. Cell growth inhibition was determined using the MTT assay. $\mathbf{b}$ THJ$16 \mathrm{~T}$ and THJ-29 T cells were infected (similar to A above) with the same as in A for 6, 12 and $24 \mathrm{~h}$. Expression levels of total and cleaved caspase-3 and PARP were analyzed by IB. $\beta$-actin was used as a control for equal loading. c 3D cultures of THJ-16 T and THJ-29 T cells were infected (similar to A above) for $48 \mathrm{~h}$ and examined for spheroid formation. Results are expressed as number of spheroids/1000 cells $\pm \mathrm{SEM},{ }^{* * *} p<0.001$. (Scale bar $\left.=100 \mu \mathrm{m}\right)$. Data are representative of the mean \pm SEM $\left.{ }^{* * *} p<0.001\right)$

THJ-16 T or THJ-29 T cells did not grow under these conditions compared to mock-infected cells (Fig. 3c).

\section{Regulating p38 MAPK signaling by rFMW/GFP}

We sought to explore the potential signaling pathways involved in rFMW/GFP-triggered oncolytic cell death in ATC cells. We examined the role of the MAPK pathway (Erk1/2, JNK and p38) as potential downstream signaling MAPK proteins, as we had previously shown these to be implicated in NDV/FMW-induced cytotoxic effects on lung cancer cells [20, 21]. p38 MAPK signaling was activated in both THJ-16 $\mathrm{T}$ and THJ-29 $\mathrm{T}$ cells upon rFMW/GFP infection at $12 \mathrm{hpi}$ and in THJ-29 T cells at 24 hpi. While phosphorylation of either Erk $1 / 2$ or JNK was not significantly altered in either cell line (Fig. 4a). In addition, we examined other critical pathways such as Akt and p53 signaling which are generally involved in cell survival and apoptosis in THJ-16 T and THJ-29 T cells infected with rFMW/GFP. As shown in the Additional file 1: Figure S1, total AKT level and p53 level were all downregulated after a 24-h infection with rFMW/GFP, suggesting that both Akt and p53 signaling might play a role in the antitumor effects by rFMW/ GFP. But change in p-Akt (S473) was not consistent between the two cell lines upon infection with rFMW/ GFP (Additional file 1: Figure S1). To investigate the role of p38 MAPK pathway in rFMW/GFP-induced cell death in ATC cells, the specific p38 MAPK inhibitor, SB203580, was added to THJ-16 T and THJ-29 T cells $30 \mathrm{~min}$ prior to virus infection. Treatment with SB203580 significantly reduced rFMW/GFP-induced cell death in both cell lines at 24 hpi. Compared to mock-infected controls (virus only) (Fig. 4b). Inhibition of p38 MAPK activity by SB203580 decreased rFMW/ GFP-induced cleavage of caspase- 3 and PARP in THJ-16 $\mathrm{T}$ and THJ-29 $\mathrm{T}$ cells (Fig. 4c). These data suggest a role for p38 MAPK in rFMW/GFP-induced oncolysis of ATC cells.

\section{Antitumor effects of rFMW/GFP in mice bearing $26 \mathrm{~T}$ - deprived tumors}

The oncolytic effects of rFMW/GFP and its parent virus NDV/FMW were investigated in mice bearing tumors derived from THJ-16 T cells. The design of these in vivo experiments were based on previous studies from our lab and others [21, 22, 24, 26, 27]. Tumor sections were examined by H\&E staining and TUNEL assay. H\&E staining of tumors treated with rFMW/GFP showed 


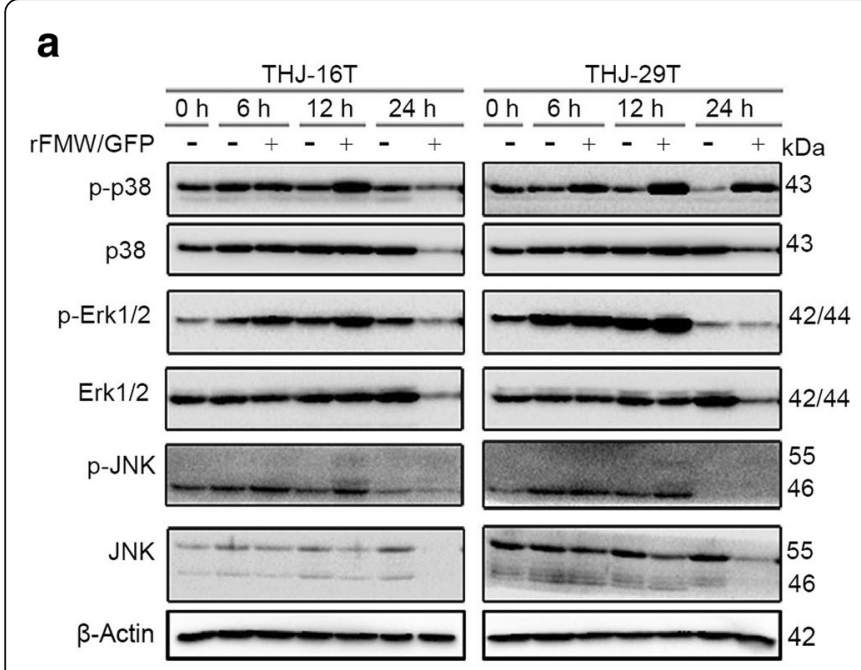

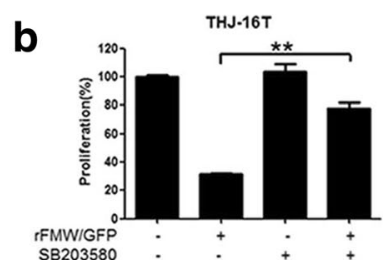

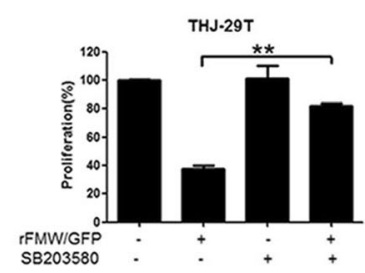

C
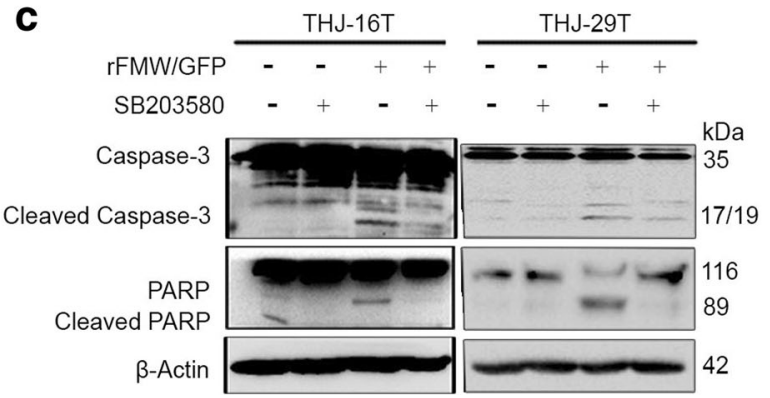

Fig. 4 Signaling pathways targeted by rFMW/GFP in ATC cell lines. a THJ-16 T and THJ-29 T cells were infected with rFMW/GFP for 6, 12 and $24 \mathrm{~h}$. Protein levels of p-p38, total p38, p-Erk1/2, total Erk1/2, p-JNK and total JNK were analyzed by immunoblotting (IB). $\beta$-actin was used as a control for equal loading. b THJ-16 T and THJ-29 T cells were infected with vehicle or $10 \mathrm{MOI}$ rFMW/GFP following pre-treatment with the p38 MAPK inhibitor, SB203580 (10 $\mu \mathrm{M})$. Cell growth inhibition was determined using the MTT assay. Data are presented as the mean \pm SEM, 0.001 $<{ }^{* *} p<0.005$. $c$ Protein expression of total and cleaved caspase-3 and PARP was examined by IB

characteristic apoptotic cells (Fig. 5a). In addition, the TUNEL assay revealed pyknotic chromatin in virusinoculated tumors (Fig. 5a). In contrast, fewer necrotic and apoptotic cells were detected in PBS-treated controls. Interestingly, immunoblotting analysis of tumor lysates demonstrated GFP and HN expression in rFMW/ GFP-inoculated tumors but not in PBS-treated tumors (Fig. 5b). Moreover, viruses isolated from tumors infected with rFMW/GFP, was shown to be infectious (data not shown), indicating that $\mathrm{rFMW/GFP} \mathrm{replicated}$ in virus-treated tumors.

Consistent with these in vivo data, significant tumor regression was observed in mice inoculated with either rFMW/GFP or parent virus compared to control groups (Fig. 5c). However, there was no significant difference in tumor growth inhibition between rFMW/GFP and parent virus. Non-tumor-bearing mice injected with either rFMW/GFP or parent virus survived and remained healthy during the course of this in vivo study.

\section{Discussion}

Although oncolytic NDV is emerging as a novel cancer therapeutic approach in the treatment of a variety of cancer types, including thyroid cancer [28], only one early report by Zamarin et al. showed NDV as an effective oncolytic agent against thyroid cancer cell lines in an in vitro study [18]. In addition, no clinical trial has been initiated with oncolytic NDV for thyroid cancer. Therefore, to our knowledge; this is the first report demonstrating that oncolytic NDV targets ATC in vitro and in vivo. We showed that the NDV/FMW strain and its derived recombinant expressing GFP, rFMW/GFP, induced cytotoxicity in ATC cells in both 2D and 3D cultures and in mice bearing ATC cell-derived tumors. Thus, our study suggests the use of oncolytic NDV as a promising therapeutic strategy for ATC.

To better track oncolytic NDV in vitro and in vivo, several oncolytic NDV strains such as D90, F3aa and Italien, have been engineered to express GFP [26, 27, 29-31]. In our previous study, the oncolytic NDV strain FMW was used as a vector to express apoptin to enhance the effects of NDV/FMW in cancer cells [24]. In the present study, the GFP gene was inserted into the genome of NDV/FMW and the resultant virus, rFMW/ GFP, replicated robustly in ATC cells as did its parent virus. Furthermore, GFP expression was observed in rFMW/GFP-infected ATC cell lines and in tumor sections from mice bearing ATC cell-derived tumors, indicating that $\mathrm{rFMW/GFP}$ can be used as a reporter virus to probe the infection process in vitro and in vivo. Moreover, analysis of the distribution of $\mathrm{rFMW/GFP}$ indicated that expression of GFP protein was detected in lung and spleen of mice intravenously injected with rFMW/GFP, in line with a previous study by Bian et al. in mice intravenously injected with the recombinant NDV strain, NDFL-EGFP [32]. Interestingly, in nonhuman primates, intravenous injection with oncolytic NDV resulted in the accumulation of the viral RNA in the respiratory tract, spleen and liver [33]. Together, our data add further knowledge to the current understanding 


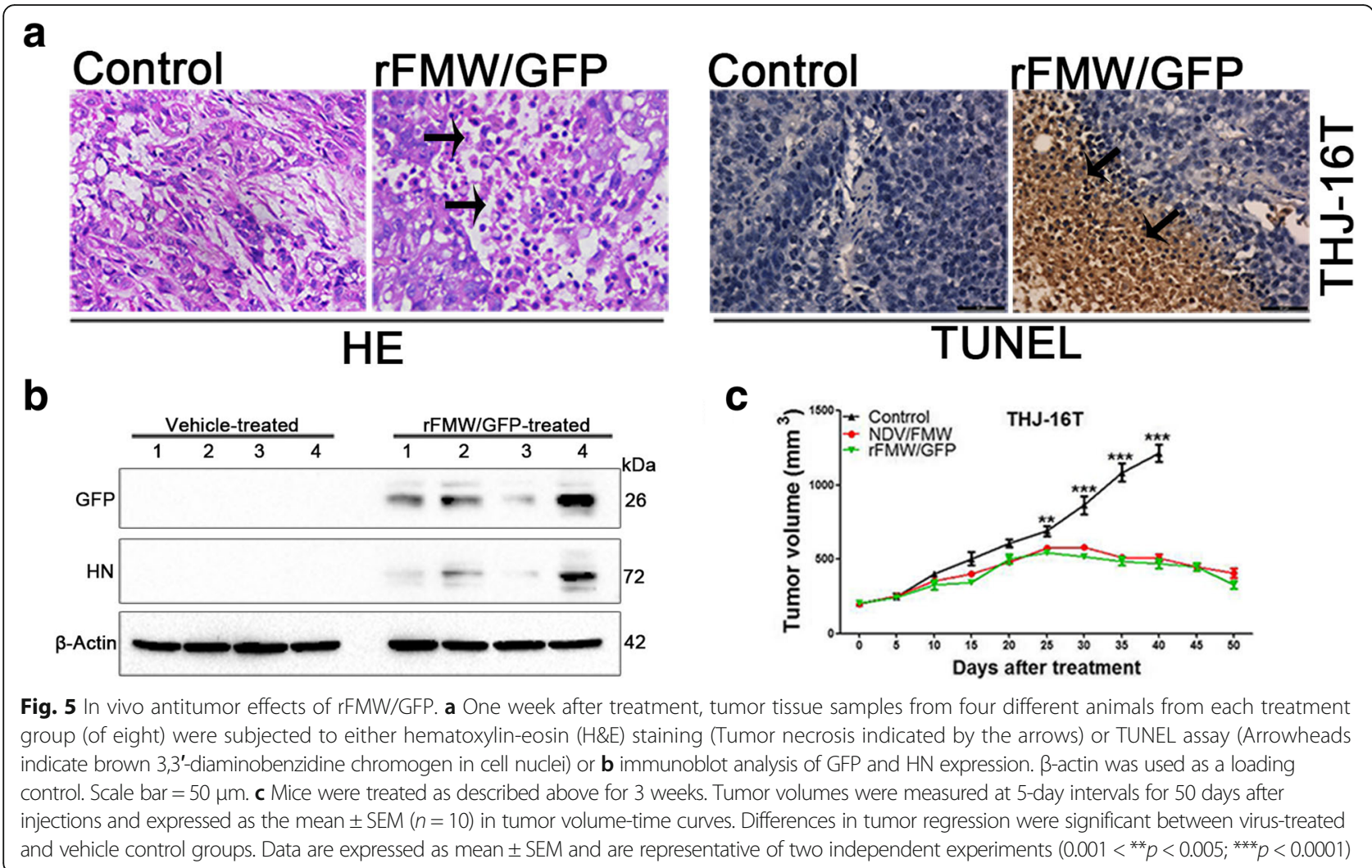

of the preclinical efficacy of rFMW/GFP in thyroid cancer cells, in addition to the administration of oncolytic NDV in animal models.

Our previous studies have shown that NDV/FMW induces apoptosis in a variety of cancer cells, during which the MAPK pathways were disturbed [20-23]. Analysis of the signaling pathway involved in $\mathrm{rFMW} /$ GFP-induced apoptosis revealed that p38MAPK, but not Erk1/2 or JNK, was activated in infected ATC cells. Furthermore, inactivation of p38MAPK activity attenuated the cytotoxic effects of rFMW/GFP on ATC cells, supporting a role of $\mathrm{p} 38$ MAPK in $\mathrm{rFMW} / \mathrm{GFP}$-induced oncolytic activity in thyroid cancer cells. These data together with our previous observations that p38 MAPK plays a role in NDV/FMW-triggered apoptosis in lung cancer cells $[20,21]$, highlight that p38 MAPK plays a role in the induction of apoptosis by oncolytic NDV in a variety of cancer types.

In summary, we present evidence showing that both the recombinant reporter virus $\mathrm{rFMW/GFP}$ and its parent virus NDV/FMW display oncolytic activities in ATC cells in vitro and in vivo. Furthermore, rFMW/GFP will be an important tool for tracing the efficacy of NDV/FMW in target cancer cells and for further elucidating the mechanism(s) by which NDV/FMW induces oncolytic cell death.

\section{Conclusions}

In the present study, we identified recombinant reporter virus rFMW/GFP display oncolytic activities in ATC cells via p38 MAPK signaling pathway and represent a novel potential therapeutic strategy for ATC.

\section{Additional file}

Additional file 1: Figure S1. Akt and p53 signaling in the antitumor effects by rFMW/GFP. THJ-16 T and THJ-29 T cells were infected with rFMW/GFP for 6, 12 and $24 \mathrm{~h}$. Protein levels of p-Akt (S473), total Akt and $\mathrm{p}-53$ were analyzed by immunoblotting (IB). $\beta$-Actin was used as a control for equal loading. (JPG $100 \mathrm{~kb}$ )

\section{Abbreviations}

3D: Three-dimensional; ATC: Anaplastic thyroid cancer; bFGF: Basic fibroblast growth factor; BSA: Bovine Serum Albumin; EGF: Epidermal growth factor; FBS: Fetal bovine serum; GFP: Green fluorescent protein; MTT: 3-(4, 5dimethylthiazol-2-yl)-2, 5-diphenyltetrazolium bromide; NDV: Newcastle disease virus; OV: Oncolytic virus; PFA: Paraformaldehyde

\section{Acknowledgements}

This work was supported by the National Science Foundation of China (Grant numbers: 81372471 to Songshu Meng, 81502674 to Ke Jiang, 31530074 to Chan Ding) and the National Science Foundation of Liaoning Province (Grant number: 2015020655 to Guirong Zhang).

\section{Funding}

This work was supported by the National Science Foundation of China (Grant numbers: 81372471 to Songshu Meng, 81502674 to Ke Jiang, 31530074 to Chan Ding) and the National Science Foundation of Liaoning 
Province (Grant number: 2015020655 to Guirong Zhang). The funding bodies have no role in the design of the study and collection, analysis, and interpretation of data and in writing the manuscript.

\section{Availability of data and materials}

Any material described in this publication can be requested directly from the corresponding author, Songshu Meng.

\section{Authors' contributions}

$\mathrm{SM}, \mathrm{CD}, \mathrm{GZ}, \mathrm{QL}$ and $\mathrm{MB}$ conceived of the study and designed the assays. KJ, CS, LK and GL performed the major experiments. WC and HC took part in confocal experiments. LH and GL performed the histological analysis; $\mathrm{KJ}$ and $\mathrm{GL}$ analyzed the data and performed the statistical analysis. TY, YW and GY performed cellular experiments, animal experiments and carried out the animal experiments data analyses. SM, MB and $\mathrm{KJ}$ wrote and edited the manuscript. All authors read and approved the final manuscript.

\section{Ethics approval and consent to participate}

All animal experiments were conducted at Dalian Medical University (Dalian, China), complying with the national guidelines for the care and use of laboratory animals and were approved by the experimental animal ethics committee at Dalian Medical University.

\section{Competing interests}

The authors have declared that no competing interests exist.

\section{Publisher's Note}

Springer Nature remains neutral with regard to jurisdictional claims in published maps and institutional affiliations.

\section{Author details}

${ }^{1}$ Institute of Cancer Stem Cell, Dalian Medical University Cancer Center, Room 415, 9 Lvshun Road South, Dalian 116044, China. ${ }^{2}$ Department of Avian Infectious Diseases, Shanghai Veterinary Research Institute, Chinese Academy of Agricultural Sciences, 518 Ziyue Road, Shanghai 200241, China. ${ }^{3}$ Thoracic Oncology Research Group, Trinity Translational Medicine Institute, Trinity Centre for Health Sciences St. James's Hospital and Trinity College Dublin, Dublin, Ireland. ${ }^{4}$ Central laboratory, Liaoning Cancer Hospital and Institute, Cancer Hospital of China Medical University, 44 Xiaoheyan Road, Shenyang 110042, China. ${ }^{5}$ Laboratory Center, The Third People's Hospital of Huizhou, Affiliated Hospital Guangzhou Medical University, Huizhou 516002, China. ${ }^{6}$ Department of Dermatology of First Affiliated Hospital, Dalian Medical University, No. 222 Zhongshan Road, Dalian 116021, China.

\section{Received: 13 October 2017 Accepted: 18 May 2018}

\section{Published online: 18 July 2018}

\section{References}

1. Nagaiah G, Hossain A, Mooney CJ, Parmentier J, Remick SC. Anaplastic thyroid cancer: a review of epidemiology, pathogenesis, and treatment. J Oncol. 2011;2011:542358

2. Bernet $V$, Smallridge R. New therapeutic options for advanced forms of thyroid cancer. Expert Opin Emerg Drugs. 2014;19(2):225-41.

3. Hallden G, Portella G. Oncolytic virotherapy with modified adenoviruses and novel therapeutic targets. Expert Opin Ther Targets. 2012;16(10):945-58.

4. Libertini S, lacuzzo I, Ferraro A, Vitale M, Bifulco M, Fusco A, Portella G. Lovastatin enhances the replication of the oncolytic adenovirus d11520 and its antineoplastic activity against anaplastic thyroid carcinoma cells. Endocrinology. 2007:148(11):5186-94.

5. Libertini S, lacuzzo I, Perruolo G, Scala S, lerano C, Franco R, Hallden G, Portella G. Bevacizumab increases viral distribution in human anaplastic thyroid carcinoma xenografts and enhances the effects of E1A-defective adenovirus dl922-947. Clin Cancer Res. 2008;14(20):6505-14.

6. Libertini S, Abagnale A, Passaro C, Botta G, Barbato S, Chieffi P, Portella G. AZD1152 negatively affects the growth of anaplastic thyroid carcinoma cells and enhances the effects of oncolytic virus d1922-947. Endocr Relat Cancer. 2011;18(1):129-41.

7. Passaro C, Volpe M, Botta G, Scamardella E, Perruolo G, Gillespie D, Libertini S, Portella G. PARP inhibitor olaparib increases the oncolytic activity of dl922-947 in in vitro and in vivo model of anaplastic thyroid carcinoma. Mol Oncol. 2015;9(1):78-92
8. Passaro C, Borriello F, Vastolo V, Di Somma S, Scamardella E, Gigantino V, Franco R, Marone G, Portella G. The oncolytic virus dl922-947 reduces IL-8/ CXCL8 and MCP-1/CCL2 expression and impairs angiogenesis and macrophage infiltration in anaplastic thyroid carcinoma. Oncotarget. 2016: 7(2):1500-15.

9. Passaro C, Abagnale A, Libertini S, Volpe M, Botta G, Cella L, Pacelli R, Hallden G, Gillespie D, Portella G. Ionizing radiation enhances dl922-947mediated cell death of anaplastic thyroid carcinoma cells. Endocr Relat Cancer. 2013;20(5):633-47.

10. Reddi HV, Madde P, Reichert-Eberhardt AJ, Galanis EC, Copland JA, Mclver B, Grebe SK, Eberhardt NL. ONYX-411, a conditionally replicative oncolytic adenovirus, induces cell death in anaplastic thyroid carcinoma cell lines and suppresses the growth of xenograft tumors in nude mice. Cancer Gene Ther. 2008;15(11):750-7.

11. Gholami S, Haddad D, Chen CH, Chen NG, Zhang Q, Zanzonico PB, Szalay AA, Fong Y. Novel therapy for anaplastic thyroid carcinoma cells using an oncolytic vaccinia virus carrying the human sodium iodide symporter. Surgery. 2011;150(6):1040-7.

12. Mundi N, Um S, Yoo J, Rizzo G, Black M, Pinto N, Palma DA, Fung K, MacNeil D, Mymryk JS, et al. The control of anaplastic thyroid carcinoma cell lines by oncolytic poxviruses. Virus Res. 2014;190:53-9.

13. Yu Z, Eisenberg DP, Singh B, Shah JP, Fong Y, Wong RJ. Treatment of aggressive thyroid cancer with an oncolytic herpes virus. Int J Cancer. 2004; 112(3):525-32.

14. Huang YY, Yu Z, Lin SF, Li S, Fong Y, Wong RJ. Nectin-1 is a marker of thyroid cancer sensitivity to herpes oncolytic therapy. J Clin Endocrinol Metab. 2007;92(5):1965-70.

15. Lin SF, Price DL, Chen CH, Brader P, Li S, Gonzalez L, Zhang Q, Yu YA, Chen $N$, Szalay AA, et al. Oncolytic vaccinia virotherapy of anaplastic thyroid cancer in vivo. J Clin Endocrinol Metab. 2008:93(11):4403-7.

16. Price $D L$, Li $P$, Chen $C H$, Wong D, Yu Z, Chen NG, Yu YA, Szalay AA, Cappello J, Fong $Y$, et al. Silk-elastin-like protein polymer matrix for intraoperative delivery of an oncolytic vaccinia virus. Head \& neck. 2016; 38(2):237-46.

17. Reddi HV, Madde P, McDonough SJ, Trujillo MA, Morris JC 3rd, Myers RM, Peng KW, Russell SJ, Mclver B, Eberhardt NL. Preclinical efficacy of the oncolytic measles virus expressing the sodium iodide symporter in iodine non-avid anaplastic thyroid cancer: a novel therapeutic agent allowing noninvasive imaging and radioiodine therapy. Cancer Gene Ther. 2012;19(9): 659-65.

18. Zamarin D, Martinez-Sobrido L, Kelly K, Mansour M, Sheng G, Vigil A, GarciaSastre A, Palese P, Fong Y. Enhancement of oncolytic properties of recombinant Newcastle disease virus through antagonism of cellular innate immune responses. Mol Ther. 2009;17(4):697-706.

19. Ganar K, Das M, Sinha S, Kumar S. Newcastle disease virus: current status and our understanding. Virus Res. 2014;184:71-81.

20. Bian J, Wang K, Kong X, Liu H, Chen F, Hu M, Zhang X, Jiao X, Ge B, Wu Y, et al. Caspase- and p38-MAPK-dependent induction of apoptosis in A549 lung cancer cells by Newcastle disease virus. Arch Virol. 2011:156(8):1335-44.

21. Meng S, Zhou Z, Chen F, Kong X, Liu H, Jiang K, Liu W, Hu M, Zhang X,

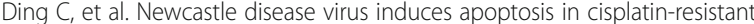
human lung adenocarcinoma A549 cells in vitro and in vivo. Cancer Lett. 2012:317(1):56-64.

22. Jiang K, Li Y, Zhu Q, Xu J, Wang Y, Deng W, Liu Q, Zhang G, Meng S. Pharmacological modulation of autophagy enhances Newcastle disease virus-mediated oncolysis in drug-resistant lung cancer cells. BMC Cancer 2014; 14:551.

23. Hu L, Sun S, Wang T, Li Y, Jiang K, Lin G, Ma Y, Barr MP, Song F, Zhang G, et al. Oncolytic Newcastle disease virus triggers cell death of lung cancer spheroids and is enhanced by pharmacological inhibition of autophagy. Am J Cancer Res. 2015;5(12):3612-23.

24. Wu Y, Zhang X, Wang X, Wang L, Hu S, Liu X, Meng S. Apoptin enhances the oncolytic properties of Newcastle disease virus. Intervirology. 2012;55(4): 276-86.

25. Marlow LA, D'Innocenzi J, Zhang Y, Rohl SD, Cooper SJ, Sebo T, Grant C, Mclver B, Kasperbauer JL, Wadsworth JT, et al. Detailed molecular fingerprinting of four new anaplastic thyroid carcinoma cell lines and their use for verification of RhoB as a molecular therapeutic target. J Clin Endocrinol Metab. 2010;95(12):5338-47.

26. Song KY, Wong J, Gonzalez L, Sheng G, Zamarin D, Fong Y. Antitumor efficacy of viral therapy using genetically engineered Newcastle disease 
virus [NDV(F3aa)-GFP] for peritoneally disseminated gastric cancer. J Mol Med. 2010;88(6):589-96.

27. Wei D, Sun N, Nan G, Wang Y, Liu HQ, Peeters B, Chen ZN, Bian H. Construction of recombinant Newcastle disease virus Italien strain for oncolytic virotherapy of tumors. Hum Gene Ther. 2012;23(7):700-10.

28. Guan M, Romano G, Coroniti R, Henderson EE. Progress in oncolytic virotherapy for the treatment of thyroid malignant neoplasm. J Exp Clin Cancer Res. 2014;33:91.

29. Silberhumer GR, Brader P, Wong J, Serganova IS, Gonen M, Gonzalez SJ, Blasberg R, Zamarin D, Fong Y. Genetically engineered oncolytic Newcastle disease virus effectively induces sustained remission of malignant pleural mesothelioma. Mol Cancer Ther. 2010;9(10):2761-9.

30. Li P, Chen CH, Li S, Givi B, Yu Z, Zamarin D, Palese P, Fong Y, Wong RJ. Therapeutic effects of a fusogenic Newcastle disease virus in treating head and neck cancer. Head \& neck. 2011;33(10):1394-9.

31. Chai Z, Zhang P, Fu F, Zhang X, Liu Y, Hu L, Li X. Oncolytic therapy of a recombinant Newcastle disease virus D90 strain for lung cancer. Virol J. 2014;11:84.

32. Bian $H$, Wilden $H$, Fournier $P$, Peeters $B$, Schirrmacher $V$. In vivo efficacy of systemic tumor targeting of a viral RNA vector with oncolytic properties using a bispecific adapter protein. Int J Oncol. 2006;29(6):1359-69.

33. Buijs $P R$, van Amerongen $G$, van Nieuwkoop $S$, Bestebroer $T M$, van Run PR, Kuiken T, Fouchier RA, van Eijck CH, van den Hoogen BG. Intravenously injected Newcastle disease virus in non-human primates is safe to use for oncolytic virotherapy. Cancer Gene Ther. 2014;21(11):463-71.

Ready to submit your research? Choose BMC and benefit from:

- fast, convenient online submission

- thorough peer review by experienced researchers in your field

- rapid publication on acceptance

- support for research data, including large and complex data types

- gold Open Access which fosters wider collaboration and increased citations

- maximum visibility for your research: over $100 \mathrm{M}$ website views per year

At BMC, research is always in progress.

Learn more biomedcentral.com/submissions 